\title{
Reliability analysis of an aging unit with a controllable repair facility activation
}

\author{
Dmitry Efrosinin, Janos Sztrik, Mais Farkhadov and Natalia Stepanova
}

\begin{abstract}
The paper utilizes the continuous-time Markov chain for modeling the processes of the gradual aging with maintenance on a finite discrete set of an intermediate failure states. The transitions occur according to the birth-and-death process and the unit fails completely after visit the last available state. The unit of a multiple and single use is studied. The switching of the repair facility is performed by a hysteresis control policy with two threshold levels for switching on/off the repair server. We provide the expressions for the stationary and non-stationary performance and reliability characteristics, solution of optimization problems and sensitivity analysis of the reliability function.
\end{abstract}

\section{Introduction}

The most technical units are continuously in operation and are subject to the gradual aging, degradation or deterioration. These processes always lead to the reduction in performance and reliability and hence must be exhaustively analyzed. Markov

Dmitry Efrosinin

Johannes Kepler University Linz, Altenbergerstrasse 69, 4040 Linz, Austria

Institute of Control Sciences, Profsoyuznaya street 65, 117997 Moscow, Russia

e-mail: dmitry.efrosinin@jku.at

Janos Sztrik

University of Debrecen, Egyetem ter, 4032 Debrecen, Hungary

e-mail: sztrik.janos@inf.unideb.hu

Mais Farkhadov

Institute of Control Sciences, Profsoyuznaya street 65, 117997 Moscow, Russia

e-mail: mais.farhadov@gmail.com

Natalia Stepanova

Altai Economics and Law Institute, Krasnoarmejskiy per 108, 656015 Barnaul, Russia

e-mail: natalia0410@ rambler.ru 
chains are widely adopted for modeling of aging processes with maintenance repair. An excellent review and contribution of the earlier papers can be found in [3], [5], [8]. The multi-state reliability models were elaborated for the aging and degradation models with gradual failures, see e.g. [2], [6, 7].

The aging process is assumed to be observable or some measure parameter can be associated with a process, e.g. signal of acoustic emission, measures of the gravimetric analysis and electromagnetic flaw detection. The hysteresis policy $\left(N_{1}, N_{2}\right)$ specifies the switching rule for the repair facility. This policy is well known in the production-inventory problems and can find also applications in maintenance of an aging unit. Here thresholds stand for the number of the passed aging states. The defined control policy can be used in a corrosion process of a unit with protective covering, in a damage process due to the fatigue crack growth, in a wear process of a tool of machine-tools, in a wear of plane bearing, in a process of discharge of an external load and so on. Two types of mathematical models are of interest. In first case the aging unit is assumed to be of a multiple use when in a complete failure state the unit can be repaired and become so good as a new one. In this case stationary characteristics are evaluated and the cost function is derived as the average reward per unit of time. In second case the aging unit operates till the first visit of the complete failure state. The expressions for the time dependent performance and reliability characteristics are derived in terms of the Laplace transform. The cost function can be represented in this case as a total average reward during a life time.

The accumulation process of the aging states can be treated as an arrival stream of the customers at the finite-population queueing system with removable server and increasing arrival rate. The Markov type models are of interest. Although the system is Markovian one only few papers deal with a removable server under $\left(N_{1}, N_{2}\right)$ policy in queues with finite population, so in contrast, performance and reliability analysis of the system with hysteresis policy combined with the finite-population queues is a new task. We derive the useful formula for computing the stationary probabilities, time dependent state probabilities, the probability density function of the remaining life time, the reliability function, the mean time to failure. Additionally a new reliability metric such as the number of switching of the repair facility is introduced as well. A cost model is derived to determine the optimal threshold policy at the average cost per unit of time for the multiple usage case and the total average cost per life time for the single usage model. Hence the results obtained in paper differ from those presented in other papers and they can be adopted for a wide variety of the Markov models with threshold-based control policies.

\section{The model description}

Assume that the aging process starts from some initial state and ends in a complete failure state. Before this process comes to the complete failure state it goes through a number $L>0$ of discrete intermediate failure states where the unit remains capable to work although with a lower efficiency. The intermediate aging states will 
be divided into two groups: the states, where the repair facility is deactivated and the transition to the previous aging state (recovering) is not possible and the states with operational repair facility, where the transitions to the neighboring states take place in both directions. The switching between the groups occurs according to the hysteresis control policy $f=\left(N_{1}, N_{2}\right)$, where $0 \leq N_{1}<N_{2}<L<\infty$. The control principle can be easily explained as follows. The first group of states includes a starting state up to the aging state $N_{2}-1$, where the transitions will be associated with a pure birth process. The further aging is accompanied by the transition to another group of states associated with a birth-and-death process. The aging process can either stay in this group until the unit will be repaired up to the state $N_{1}+1$ or reach the complete failure state, where the unit can be completely repaired or not.

Let $D(t) \in\{0,1\}$ denote the state of the server at time $t, 0$ and 1 means that the server is switched off and on, and $N(t)$ denote the number of customers in the system at time $t$. The system states at time $t$ are described by the continuous-time Markov chain which will refer to as Markov process,

$$
\{X(t)\}_{t \geq 0}=\{D(t), N(t)\}_{t \geq 0}
$$

with a state space

$$
E=\left\{x=(0, n) ; 0 \leq n \leq N_{2}-1,(1, n) ; N_{1}+1 \leq n \leq L\right\}
$$

and infinitesimal matrix $\Lambda=\left[\lambda_{x y}\right]_{x, y \in E}, \lambda_{x}=-\lambda_{x x}=\sum_{y \neq x} \lambda_{x y}$, where $\lambda_{x y}=\lambda_{x y}(f)$ depends on the switching hysteresis policy $f=\left(N_{1}, N_{2}\right)$.

\section{Stationary probabilities and average reward}

According to above description $\{X(t)\}_{t \geq 0}$ is an irreducible Markov process where

$$
\pi=\left(\pi_{(0,0)}, \pi_{(0,1)} \ldots, \pi_{\left(0, N_{2}-1\right)}, \pi_{\left(1, N_{1}+1\right)}, \pi_{\left(1, N_{1}+2\right)}, \ldots, \pi_{(1, L)}\right)
$$

is a stationary probability row-vector for the policy $f=\left(N_{1}, N_{2}\right)$. The system of balance equations is of the form,

$$
\begin{aligned}
(n+1) \lambda \pi_{(0, n)} & =n \lambda \pi_{(0, n-1)}, 0 \leq n \leq N_{2}-1, n \neq N_{1}, \\
\left(N_{1}+1\right) \lambda \pi_{\left(0, N_{1}\right)} & =N_{1} \lambda \pi_{\left(0, N_{1}-1\right)}+\mu \pi_{\left(1, N_{1}+1\right)}, \\
\left(\left(N_{2}+1\right) \lambda+\mu\right) \pi_{\left(1, N_{1}+1\right)} & =\mu \pi_{\left(1, N_{1}+2\right)}, \\
((n+1) \lambda+\mu) \pi_{(1, n)} & =n \lambda \pi_{(1, n-1)}+\mu \pi_{(1, n+1)}, N_{1}+2 \leq n \leq L-1, n \neq N_{2}, \\
\left(\left(N_{2}+1\right) \lambda+\mu\right) \pi_{\left(1, N_{2}\right)} & =N_{2} \lambda\left(\pi_{\left(1, N_{2}-1\right)}+\pi_{\left(0, N_{2}-1\right)}\right)+\mu \pi_{\left(1, N_{2}+1\right)}, \\
\mu \pi_{(1, L)} & =(L-1) \lambda \pi_{(1, L-1)} .
\end{aligned}
$$


Since the set $E$ is finite, $\pi$ exists and satisfies the system $\pi \Lambda=\mathbf{0}, \pi \mathbf{e}=1$. Define the following cost structure:

$c_{1}$ - the reward per unit of time for each remaining failure state if server is off, $c_{2}$ - the reward per unit of time for each remaining failure state if server is on, $(n+1) c_{3}$ - fixed costs per switching on of the repair facility at aging state $n$, $n c_{4}$ - fixed costs per switching off of the repair facility at aging state $n$, $c_{5}$ - the repair costs per unit of time in a complete failure state $x=(1, L)$.

Now we can formulate optimization problem: Find an optimal policy $f^{*}=$ $\left(N_{1}^{*}, N_{2}^{*}\right)$ to maximize the average reward per unit of time

$$
g^{f}=\sum_{x \in E} c(x, f) \pi_{x}^{f}, \text { where }
$$

$c(x, f)=c(x)-\sum_{y \neq x} \lambda_{x y}(f) c_{x y}(f)$-immediate cost in state $x$ under policy $f$,

$c(x)=c_{1} \sum_{n=0}^{N_{2}-1}(L-n) 1_{\{x=(0, n)\}}+c_{2} \sum_{n=N_{1}+1}^{L-1}(L-n) 1_{\{x=(1, n)\}}-$

reward per unit of time when the process is in state $x \in E$,

$\sum_{y \neq x} \lambda_{x y}(f) c_{x y}(f)=\left(N_{1}+1\right) c_{3} \mu 1_{\left\{x=\left(1, N_{1}+1\right)\right\}}+N_{2}^{2} c_{4} \lambda 1_{\left\{x=\left(0, N_{2}-1\right)\right\}}+c_{5} 1_{\{x=(1, L)\}}-$

fixed cost incurred each time when the process jumps from $x$ to $y$.

Denote by

$$
\begin{aligned}
& C_{n}^{l}=\prod_{k=n}^{l} \tau_{k}, \tau_{N_{1}+1}=\frac{\mu}{\left(N_{1}+2\right) \lambda+\mu}, \\
& \tau_{k}=\frac{\mu}{(k+1) \lambda+\mu-k \lambda \tau_{k-1}}, N_{1}+2 \leq k \leq L-1, k \neq N_{2}, \\
& \tau_{N_{2}}=\frac{\mu}{\left(N_{2}+1\right) \lambda+\mu-N_{2} \lambda \tau_{N_{2}-1}-\mu C_{N_{1}+1}^{N_{2}-1}} .
\end{aligned}
$$

Theorem 1. The average reward per unit of time has the form

$$
\begin{aligned}
g\left(N_{1}, N_{2}\right) & =c_{1} \sum_{n=0}^{N_{2}-1}(L-n) \pi_{(0, n)}+c_{2} \sum_{n=N_{1}+1}^{L-1}(L-n) \pi_{(1, n)} \\
& -\left(\left(N_{1}+1\right) c_{3} \mu \pi_{\left(1, N_{1}+1\right)}+\left(N_{2}-1\right) N_{2} c_{4} \lambda \pi_{\left(0, N_{2}-1\right)}+c_{5} \pi_{(1, L)}\right)
\end{aligned}
$$

where $\pi_{x}, x \in E$, satisfy the explicit expressions,

$$
\pi_{(1, L)}=\left[1+\frac{\mu}{\lambda} C_{N_{1}+1}^{L-1} \sum_{n=N_{1}}^{N_{2}-1} \prod_{i=N_{1}}^{n} \frac{1}{i+1}+\sum_{n=N_{1}+1}^{L-1} C_{n}^{L-1}\right]^{-1}
$$




$$
\begin{aligned}
& \pi_{(0, n)}=\frac{\mu}{\lambda} C_{N_{1}+1}^{L-1} \prod_{i=N_{1}}^{n} \frac{1}{i+1} \pi_{(1, L)}, N_{1} \leq n \leq N_{2}-1, \\
& \pi_{(1, n)}=C_{n}^{L-1} \pi_{(1, L)}, N_{1}+1 \leq n \leq L-1 .
\end{aligned}
$$

Proof. The statement follows by solving recursively the system of balance equations for the stationary state probabilities taking into account that for transient states $\pi_{(0, n)}=0,0 \leq n \leq N_{1}-1$.

Our aim is to find the optimal policy $f^{*}=\left(N_{1}^{*}, N_{2}^{*}\right)$ such that

$$
\begin{aligned}
& g\left(f^{*}\right)=\min _{f} g(f), \\
& \text { subject to } f=\left(N_{1}, N_{2}\right), 0 \leq N_{1}<N_{2}<L .
\end{aligned}
$$

It is also possible formulate an optimization problem with the aim to find the joint optimal value $\left(f^{*}, \mu^{*}\right)$. Mathematically it can be described by

$$
\begin{aligned}
& g\left(f^{*}, \mu^{*}\right)=\min _{f, \mu} g(f, \mu) \\
& \text { subject to } 0 \leq N_{1}<N_{2}<L, 0<\mu<\mu^{U},
\end{aligned}
$$

where $\mu^{U}$ is predefined upper bound. The function $g$ is nonlinear and quite complex in order to solve the optimization problem analytically. To find a discrete optimal vector $f^{*}$ for the fixed parameters a direct search method can be applied. For the joint values $\left(f^{*}, \mu^{*}\right)$ a method for numerical solution of the cost optimization problem can be used. It uses the principles of the a quasi-Newton method, see. e.g. [1].

\section{Reliability analysis during the life time}

Assume that at time $t=0$ the system starts from initial state $x=(0,0)$ and the complete failure state $(1, L)$ will be the absorbing one. Denote by $T$ the life time of the system or the time to absorption, i.e. $T=\inf \{t: X(t)=(1, L)\}$. Here we analyze the system during the time $T$. The transient Markov process $\hat{X}(t)$, which describes the system states at time $t$, has the same state space $E$ and almost the same infinitesimal matrix $\Lambda$ as (1) with one exception that in latter case there is no transition from $(1, L)$ to $(1, L-1)$. For the time dependent state probabilities

$$
\begin{aligned}
& \pi_{(0, n)}(t)=\mathbb{P}[X(t)=(0, n), t<T], 0 \leq n \leq N_{2}-1 \\
& \pi_{(1, n)}(t)=\mathbb{P}[X(t)=(1, n), t<T], N_{1}+1 \leq n \leq L,
\end{aligned}
$$

referring the state transition rate diagram we can write down the corresponding Kolmogorov differential equations (KDE), $\pi^{\prime}(t)=\pi(t) \Lambda$ with initial condition $\pi_{(0,0)}(0)=1, \pi_{x}(0)=0, x \neq(0,0)$. Applying the Laplace transforms (LT) $\tilde{\pi}_{(d, n)}(s)=\int_{0}^{\infty} e^{-s t} \pi_{(d, n)}(t) d t, \operatorname{Re}[s]>0$, we get the system of equations, which can 
be rewritten in matrix form

$$
\tilde{\pi}(s) \Lambda(s)=\pi(0),
$$

where $\tilde{\pi}(s)=\left(\tilde{\pi}_{(0,0)}(s), \ldots, \tilde{\pi}_{\left(0, N_{2}-1\right)}(s), \tilde{\pi}_{\left(1, N_{1}+1\right)}(s), \ldots, \tilde{\pi}_{(1, L)}(s)\right), \Lambda(s)=s I-\Lambda$ is a $\left(L+N_{2}-N_{1}\right) \times\left(L+N_{2}-N_{1}\right)$ matrix, $I$ is the identity matrix of the appropriate size, $\pi(0)=(1,0, \ldots, 0)$ - initial probability vector. It can be shown that for the Markov process $\{\hat{X}(t)\}_{t \geq 0}$ with an absorption the total average reward is equal to

$$
g^{f}=\sum_{x \in E} c(x, f) \int_{0}^{T} \pi_{x}(u) d u=\sum_{x \in E} c(x, f) \tilde{\pi}_{x}(0) .
$$

Denote by

$$
\begin{aligned}
& B_{n}^{l}(s)=\prod_{k=n}^{l} \rho_{k}(s), \rho_{0}(s)=\frac{1}{s+\lambda}, \rho_{k}(s)=\frac{k \lambda}{s+(k+1) \lambda}, \\
& C_{n}^{l}(s)=\prod_{k=n}^{l} \tau_{k}(s), \tau_{N_{1}}(s)=\frac{\mu}{s+\left(N_{1}+1\right) \lambda}, \tau_{N_{1}+1}(s)=\frac{\mu}{s+\left(N_{1}+2\right) \lambda+\mu}, \\
& \tau_{N_{2}}(s)=\frac{\mu}{s+\left(N_{2}+1\right) \lambda+\mu-N_{2} \lambda \tau_{N_{2}-1}(s)-N_{2} \lambda B_{N_{1}+1}^{N_{2}-1}(s) C_{N_{1}}^{N_{2}-1}(s)}, \\
& \tau_{k}(s)=\frac{\mu}{s+(k+1) \lambda+\mu-k \lambda \tau_{k-1}(s)}, N_{1}+2 \leq k \leq L-1, k \neq N_{2}, \\
& v_{N_{2}}(s)=\frac{N_{2} \lambda B_{0}^{N_{2}-1}(s)}{s+\left(N_{2}+1\right) \lambda+\mu-N_{2} \lambda \tau_{N_{2}-1}(s)-N_{2} \lambda B_{N_{1}+1}^{N_{2}-1}(s) C_{N_{1}}^{N_{2}-1}(s)}, \\
& v_{k}(s)=\frac{k \lambda v_{k-1}(s)}{s+(k+1) \lambda+\mu-k \lambda \tau_{k-1}(s)}, N_{2}+1 \leq k \leq L-1, \\
& v_{L}(s)=\frac{L \lambda v_{L-1}(s)}{s-L \lambda \tau_{L-1}(s)} .
\end{aligned}
$$

Theorem 2. The total average reward during the time $T$ has the form

$$
\begin{aligned}
g\left(N_{1}, N_{2}\right) & =c_{1} \sum_{n=0}^{N_{2}-1}(L-n) \tilde{\pi}_{(0, n)}(0)+c_{2} \sum_{n=N_{1}+1}^{L-1}(L-n) \tilde{\pi}_{(1, n)}(0) \\
& -\left(c c_{3} \mu \tilde{\pi}_{\left(1, N_{1}+1\right)}(0)+c_{4} N_{2} \lambda \tilde{\pi}_{\left(0, N_{2}-1\right)}(0)\right), \text { where } \\
\tilde{\pi}_{(0, n)}(s) & =B_{0}^{n}(s), 0 \leq n \leq N_{1}-1, \\
\tilde{\pi}_{(0, n)}(s) & =B_{0}^{n}(s)+\tau_{N_{1}}(s) B_{N_{1}+1}^{n}(s) \tilde{\pi}_{\left(1, N_{1}+1\right)}(s), N_{1} \leq n \leq N_{2}-1, \\
\tilde{\pi}_{(1, n)}(s) & =\sum_{i=0}^{L-N_{2}} C_{n}^{N_{2}+i-1}(s) v_{N_{2}+i}(s), N_{1}+1 \leq n \leq N_{2}-1,
\end{aligned}
$$




$$
\tilde{\pi}_{(1, n)}(s)=\sum_{i=0}^{L-n} C_{n}^{n+i-1}(s) v_{n+i}(s), N_{2} \leq n \leq L
$$

is a solution of the system of KDEs in terms of the LT.

Proof. The statement follows by solving recursively the system of KDEs in terms of the LT.

Denote by

$T_{y x}$ - time spent in $x \in E$ starting from $y$,

$T_{y}=\sum_{x \in E} T_{y x}$ - time to absorption from $y$ (residual life time).

For the initial distribution $\pi(0)$ over $E$, define a ratio of means distribution by

$$
p_{x}=\frac{\sum_{y \in E} \pi_{y}(0) \mathbb{E}\left[T_{y x}\right]}{\sum_{y \in E} \pi_{y}(0) \mathbb{E}\left[T_{y}\right]}=\frac{\sum_{y \in E} \int_{0}^{\infty} \pi_{y}(0) p_{y x}(t) d t}{\sum_{y \in E} \int_{0}^{\infty} \pi_{y}(0)\left(1-p_{y(1, L)}(t)\right) d t},
$$

where $p_{y x}(t)$ is a transition probability from state $y$ to state $x$ in time $t$ of the absorbing Markov chain $\{\hat{X}(t)\}_{t \geq 0}$.

Remark 1. The reward function $g\left(N_{1}, N_{2}\right)$ can be evaluated with respect to the ratio of means distribution $p_{(d, n)}$ depending on $\pi(0)$,

$$
p_{(d, n)}=\frac{\tilde{\pi}_{(d, n)}(0)}{\sum_{n=0}^{N_{2}-1} \tilde{\pi}_{(0, n)}(0)+\sum_{n=N_{1}+1}^{L-1} \tilde{\pi}_{(1, n)}(0)} .
$$

In this case the optimal policy $\left(N_{1}, N_{2}\right)$ can differ from that which minimizes the total reward.

\section{Reliability function and evaluation methods}

The system reliability function is defined by

$$
R(t)=\mathbb{P}[T>t]=1-\pi_{(1, L)}(t), t \geq 0 .
$$

If $\tilde{R}(s)=\int_{0}^{\infty} e^{-s t} R(t) d t, \operatorname{Re}[s]>0$, then it follows

$$
\tilde{R}(s)=\frac{1}{s}-\tilde{\pi}_{(1, L)}(s) .
$$

Method 1 - Direct solution of the KDE.

The LT $\tilde{\pi}_{(1, L)}(s)$ can be calculated from the system of KDE,

$$
\tilde{\pi}_{(1, L)}(s)=v_{L}(s) .
$$


The inversion of the Laplace transform completes the evaluation.

Method 2 - Solution of the system of KDE using Cramer's rule.

The LT $\tilde{\pi}_{(1, L)}(s)$ can be evaluated also by solving the system

$$
\begin{aligned}
& \tilde{\pi}(s)(s I-\Lambda)=\pi(0), \\
& \pi(0)=(1,0, \ldots, 0)
\end{aligned}
$$

using the Cramer's rule

$$
\tilde{\pi}_{(1, L)}(s)=\frac{\left|\Lambda_{L+N_{2}-N_{1}}(s)\right|}{|\Lambda(s)|} \text {, where }
$$

$|\Lambda(s)|$ - the determinant of the matrix $\Lambda(s)$,

$\left|\Lambda_{L+N_{2}-N_{1}}(s)\right|-$ the determinant obtained by replacing the $\left(L+N_{2}-N_{1}\right)$ th raw of $\Lambda(s)$ by the initial vector $\pi(0)$.

Theorem 3. The determinants $\left|\Lambda_{L+N_{2}-N_{1}}(s)\right|$ and $|\Lambda(s)|$ are of the form

$$
\begin{aligned}
& \left|\Lambda_{L+N_{2}-N_{1}}(s)\right|=(-1)^{L} \lambda^{L} L !\left|\Delta_{N_{1}+2, N_{2}+1}(s)\right|, \\
& |\Lambda(s)|=s \prod_{i=1}^{N_{1}}(s+i \lambda)\left[\prod_{i=N_{1}+1}^{N_{2}}(s+i \lambda)\left|\Delta_{N_{1}+2, L}(s)\right|\right. \\
& \left.+\frac{N_{2} !(\lambda \mu)^{N_{2}-N_{1}}}{N_{1} !}\left|\Delta_{N_{2}+2, L}(s)\right|\right], \text { where } \\
& \left|\Delta_{k, l}(s)\right|=\prod_{i=k}^{l} a_{i}\left[1+\sum_{r=1}^{\left\lfloor\frac{l-k+1}{2}\right\rfloor} i_{0}, \ldots, i_{r-1} \in S_{r}(2, l-k+1) \prod_{j=0}^{r} \frac{\mu b_{k+i_{j}-2}}{a_{k+i_{j}-2} a_{k+i_{j}-1}}\right], \\
& S_{r}(2, n)= \begin{cases}\{2, \ldots, n\} & n \geq 2, r=1, \\
\left\{\left(i_{1}, \ldots, i_{r}\right): i_{j} \in\{2, \ldots, n\}, i_{j}-i_{j-1} \geq 2\right\}, & n \geq 4,2 \leq r \leq\left\lfloor\frac{n}{2}\right\rfloor,\end{cases}
\end{aligned}
$$

$a_{l}=(s+l \lambda+\mu), b_{l}=-l \lambda, \Delta_{k, l}(s)$-tridiagonal matrix with upper, main and lower diagonals given by $\left(b_{k}, \ldots, b_{l-1}\right),\left(a_{k}, \ldots, a_{l}\right),(-\mu, \ldots,-\mu)$.

Proof. The result can be easily obtained using the definition of the tridiagonal matrix and its properties, $\left|\Delta_{k, l}(s)\right|=a_{l}\left|\Delta_{k, l-1}(s)\right|+b_{l-1} \mu\left|\Delta_{k, l-2}(s)\right|,\left|\Delta_{k, k-1}(s)\right|=$ $1,\left|\Delta_{k, k-2}(s)\right|=0$. Solving this difference equation as proposed in [4], we get the explicit expression for the determinant $\left|\Delta_{k, l}(s)\right|$.

Theorem 4. The reliability function $R(t)$ satisfies the relation

$$
\begin{aligned}
R(t) & =-\sum_{k=1}^{l} A_{l} e^{-s_{k} t}+\sum_{k=1}^{m} e^{-\operatorname{Re}\left[s_{l+k}\right] t}\left[B_{k} \cos \left(\operatorname{Im}\left[s_{l+k}\right] t\right)\right. \\
& \left.+\frac{C_{k}-B_{k} \operatorname{Re}\left[s_{l+k}\right]}{\operatorname{Im}\left[s_{l+k}\right]} \sin \left(\operatorname{Im}\left[s_{l+k}\right] t\right)\right], t \geq 0, \text { where }
\end{aligned}
$$




$$
\begin{aligned}
& A_{0}=\left.\frac{s\left|\Lambda_{L+N_{2}-N_{1}}(0)\right|}{|\Lambda(s)|}\right|_{s=0}=1, \\
& A_{n}=\left.\frac{\left(s+s_{n}\right)\left|\Lambda_{L+N_{2}-N_{1}}\left(-s_{n}\right)\right|}{|\Lambda(s)|}\right|_{s=-s_{n}}, 1 \leq n \leq l, \\
& B_{n} s_{l+n}+C_{n}= \\
& \left.\frac{\left(s+\left(s_{l+n}+\bar{s}_{l+n}\right) s+s_{l+n} \bar{s}_{l+n}\right)\left|\Lambda_{L+N_{2}-N_{1}}\left(-s_{l+n}\right)\right|}{|\Lambda(s)|}\right|_{s=-s_{l+n}}, 1 \leq n \leq m,
\end{aligned}
$$

$s_{n}=n \lambda, 1 \leq n \leq N_{1}, s_{n}=n \lambda+\mu, N_{2}+2 \leq n \leq L$ and other eigenvalues are the solutions of $|\Delta(s)|=0$.

Proof. The determinant $|\Lambda(s)|$ can be factorized,

$$
|\Lambda(s)|=s \prod_{k=1}^{l}\left(s+s_{k}\right) \prod_{k=1}^{m}\left(s^{2}+\left(s_{l+k}+\bar{s}_{l+k}\right) s+s_{l+k} \bar{s}_{l+k}\right),
$$

where $s_{0}=0$ and $s_{1}, s_{2}, \ldots, s_{l}$ are the possible $l$ real distinct eigenvalues, $\left(s_{l+1}, \bar{s}_{l+1}\right),\left(s_{l+2}, \bar{s}_{l+2}\right), \ldots$, $\left(s_{l+m}, \bar{s}_{l+m}\right)$ are the $m$ pairs of distinct conjugate complex eigenvalues obtained from

$$
|\Lambda(s)|=|\Lambda-s I|=0
$$

Due to the partial fraction expansion,

$$
\tilde{\pi}_{(1, L)}(s)=\sum_{k=0}^{l} \frac{A_{k}}{s+s_{k}}+\sum_{k=1}^{m} \frac{B_{k} s+C_{k}}{s^{2}+\left(s_{l+k}+\bar{s}_{l+k}\right) s+s_{l+k} \bar{s}_{l+k}} .
$$

The constants $A_{k}, 0 \leq k \leq l$, are the real numbers, which by equating the coefficients can be obtained in form (14). Note that $A_{0}=\lim _{t \rightarrow \infty} \pi_{(1, L)}(t)=1$. Together with (9) the inverse LT implies the explicit relation (13).

\section{Method 3 - The remaining life time.}

Denote by

$T_{(d, n)}=T \mid X(0)=(d, n)$ - the remaining life time given $(d, n) \in E$,

$r_{x}(t)=\frac{\mathbb{P}\left[T_{x} \in[t, t+d t)\right]}{d t}-$ probability density function,

$\tilde{r}_{x}(s)=\int_{0}^{\infty} e^{-s t} r_{x}(t) d t-$ LT of the density function $r_{x}(t)$.

The LT $\tilde{R}(s)$ can be evaluated by

$$
\tilde{R}(s)=\frac{1}{s}\left[1-\tilde{r}_{(0,0)}(s)\right] .
$$

Note that $\tilde{\pi}_{(1, L)}(s)=\frac{L \lambda}{s} \tilde{\pi}_{(1, L-1)}(s)$. It follows then that

$$
\tilde{r}_{(0,0)}(s)=L \lambda \tilde{\pi}_{(1, L-1)}(s) .
$$

The conditional LT $\tilde{r}_{x}(s)$ can be evaluated directly as well. Define 


$$
\begin{aligned}
& B_{n}^{l}(s)=\prod_{k=n}^{l} \rho_{k}(s), \rho_{k}(s)=\frac{k \lambda}{s+k \lambda}, \\
& C_{n}^{l}(s)=\prod_{k=n}^{l} \tau_{k}(s), \tau_{N_{1}+1}(s)=\frac{\left(N_{1}+2\right) \lambda}{s+\left(N_{1}+2\right) \lambda+\mu}, v_{N_{1}+1}(s)=\frac{\mu B_{N_{1}+1}^{N_{2}}(s)}{s+\left(N_{1}+2\right) \lambda+\mu}, \\
& \tau_{k}(s)=\frac{(k+1) \lambda}{s+(k+1) \lambda+\mu-\mu \tau_{k-1}(s)}, N_{1}+2 \leq k \leq L-1, k \neq N_{2}-1, \\
& v_{k}(s)=\frac{\mu v_{k-1}(s)}{s+(k+1) \lambda+\mu-\mu \tau_{k-1}(s)}, N_{1}+2 \leq k \leq N_{2}-2, \\
& \tau_{N_{2}-1}(s)=\frac{N_{2} \lambda+\mu v_{N_{2}-2}(s)}{s+N_{2} \lambda+\mu-\mu \tau_{N_{2}-2}(s)} .
\end{aligned}
$$

Theorem 5. The LTs $\tilde{r}_{x}(s), x \in E$, are obtained by

$$
\begin{aligned}
& \tilde{r}_{(0, n)}(s)=B_{n+1}^{N_{2}}(s) C_{N_{2}}^{L-1}(s), 0 \leq n \leq N_{2}-1, \\
& \tilde{r}_{(1, n)}(s)=C_{N_{2}}^{L-1}(s) \sum_{i=0}^{N_{2}-n-1} C_{n}^{n+i-1}(s) v_{n+i}(s), N_{1}+1 \leq n \leq N_{2}-2, \\
& \tilde{r}_{(1, n)}(s)=C_{n}^{L-1}(s), N_{2}-1 \leq n \leq L-1 .
\end{aligned}
$$

Proof. Due to the Markov property $r_{x}(t)=\sum_{y \neq x} \lambda_{x y} e^{-\lambda_{x} t} * r_{y}(t)$, hence

$$
\begin{aligned}
& \tilde{r}_{x}(s)=\sum_{y \neq x} \frac{\lambda_{x y}}{s+\lambda_{x}} \tilde{r}_{y}(s), x \in E \backslash\{(1, L)\}, \\
& \tilde{r}_{(1, L)}(s)=1 .
\end{aligned}
$$

Recursively solving the last system we get the statement.

Corollary 1. The mean time to failure (MTTF) is obtained as

$$
\begin{aligned}
\mathbb{E}[T] & =\int_{0}^{\infty} R(t) d t=\lim _{s \rightarrow 0} \tilde{R}(s)=-\left.\frac{d}{d s} s \tilde{\pi}_{(1, L)}(s)\right|_{s=0}=-\left.\frac{d}{d s} \tilde{r}_{(0,0)}(s)\right|_{s=0} \\
& =\sum_{k=1}^{l} \frac{A_{k}}{s_{k}}+\sum_{k=1}^{m} \frac{C_{k}}{s_{l+k} \bar{s}_{l+k}} .
\end{aligned}
$$

Theorem 6. The mean time to failure (MTTF) is obtained as

$$
\begin{gathered}
\mathbb{E}[T]=\frac{1}{\lambda} \sum_{i=1}^{N_{2}} \frac{1}{i}+\sum_{i=0}^{L-N_{2}-1} C_{N_{2}}^{N_{2}+i-1} v_{N_{2}+i}, \text { where } \\
C_{n}^{l}=\prod_{k=n}^{l} \tau_{k}, \tau_{N_{1}+1}=\frac{\left(N_{1}+2\right) \lambda}{\left(N_{1}+2\right) \lambda+\mu}, v_{N_{1}+1}=\frac{\mu}{\left(N_{1}+2\right) \lambda+\mu}, \xi_{N_{1}+1}=v_{N_{1}+1} \sum_{i=N_{1}+1}^{N_{2}} \frac{1}{i},
\end{gathered}
$$


$\tau_{k}=\frac{(k+1) \lambda}{(k+1) \lambda+\mu-\mu \tau_{k-1}}, v_{k}=\frac{\mu v_{k-1}}{(k+1) \lambda+\mu-\mu \tau_{k-1}}, N_{1}+2 \leq k \leq L-2, k \neq N_{2}$,

$\xi_{k}=\frac{\mu \xi_{k-1}}{(k+1) \lambda+\mu-\mu \tau_{k-1}}, N_{1}+1 \leq k \leq N_{2}-1$

$\tau_{N_{2}}=\frac{\left(N_{2}+1\right) \lambda}{\left(N_{2}+1\right) \lambda+\mu-\mu\left(\tau_{N_{2}-1}+v_{N_{2}-1}\right)}, v_{N_{2}}=\frac{\mu \xi_{N_{2}-1}}{\left(N_{2}+1\right) \lambda+\mu-\mu\left(\tau_{N_{2}-1}+v_{N_{2}-1}\right)}$,

$v_{L-1}=\frac{1+\mu v_{L-2}}{L \lambda+\mu-\mu \tau_{L-2}}$.

Proof. $\bar{r}_{x}=\mathbb{E}\left[T_{x}\right]$ can be calculated by

$$
\bar{r}_{x}=\frac{1}{\lambda_{x}}\left[1+\sum_{y \neq x} \frac{\lambda_{x y}}{\lambda_{x}} \bar{r}_{y}\right]
$$

which follows by differentiating the expressions for $\tilde{r}_{x}(s)$ in point $s=0$.

Now we derive the distribution of the number of switches of the repair facility during the life time. Denote by

$K$ - the number of switches (loops) of the repair facility left up to absorption time $T$, $\psi_{(d, n)}(k)=\mathbb{P}[K=k \mid X(0)=(d, n)]$ - the probability density function (PDF), $\tilde{\psi}_{(d, n)}(z)=\sum_{k=1}^{\infty} z^{k} \psi_{(d, n)}(k),|z|<1-$ the generating function (GF).

The study of this descriptor complements the reliability analysis providing a type of a discrete counterpart of the length of $T$.

Theorem 7. The GF $\tilde{\psi}_{x}(z), x \in E$, satisfies the system for $\tilde{r}_{x}(s)$ for $s=0$, but the service rate $\mu$ in numerator of $v_{N_{1}+1}(s)$ is replaced by $z \mu$.

$$
\begin{gathered}
\tilde{\Psi}_{(0, n)}(z)=z C_{N_{2}}^{L-1}(z), 0 \leq n \leq N_{2}-1, \\
\tilde{\Psi}_{(1, n)}(z)=C_{N_{2}}^{L-1}(z) \sum_{i=0}^{N_{2}-n-1} C_{n}^{n+i-1}(z) v_{n+i}(z), N_{1}+1 \leq n \leq N_{2}-2, \\
\tilde{\Psi}_{(1, n)}(z)=C_{n}^{L-1}(z), N_{2}-1 \leq n \leq L-1, \text { where } \\
C_{n}^{l}(z)=\prod_{k=n}^{N_{2}-1} \tau_{k} 1_{\left\{l \leq N_{2}-2\right\}} \prod_{k=i+1}^{l} \tau_{k}(z) 1_{\left\{i>N_{2}-2\right\}}, \\
\tau_{N_{1}+1}=\frac{\left(N_{1}+2\right) \lambda}{\left(N_{1}+2\right) \lambda+\mu}, v_{N_{1}+1}(z)=\frac{z \mu}{\left(N_{1}+2\right) \lambda+\mu}, \\
\tau_{k}=\frac{(k+1) \lambda}{(k+1) \lambda+\mu-\mu \tau_{k-1}}, v_{k}(z)=\frac{\mu v_{k-1}(z)}{(k+1) \lambda+\mu-\mu \tau_{k-1}}, N_{1}+2 \leq k \leq N_{2}-2, \\
\tau_{N_{2}-1}(z)=\frac{N_{2} \lambda+\mu v_{N_{2}-2}(z)}{N_{2} \lambda+\mu-\mu \tau_{N_{2}-2}(z)}, \tau_{k}(z)=\frac{(k+1) \lambda}{(k+1) \lambda+\mu-\mu \tau_{k-1}(z)}, N_{2} \leq k \leq L-1 .
\end{gathered}
$$

Proof. Due to the Markov property 


$$
\psi_{x}(k)=\sum_{\substack{y \neq x \\ y \neq\left(1, N_{2}\right)}} \frac{\lambda_{x y}}{\lambda_{x}} \psi_{y}(k) 1_{\left\{x \neq\left(0, N_{2}-1\right)\right\}}+\frac{N_{2} \lambda}{\lambda_{x}} \psi_{\left(1, N_{2}\right)}(k-1) 1_{\left\{x=\left(0, N_{2}-1\right)\right\}},
$$

or in terms of the generating function

$$
\tilde{\psi}_{x}(z)=\sum_{\substack{y \neq x \\ y \neq\left(1, N_{2}\right)}} \frac{\lambda_{x y}}{\lambda_{x}} \tilde{\psi}_{y}(z) 1_{\left\{x \neq\left(0, N_{2}-1\right)\right\}}+\frac{z N_{2} \lambda}{\lambda_{x}} \tilde{\psi}_{\left(1, N_{2}\right)}(z) 1_{\left\{x=\left(0, N_{2}-1\right)\right\}} .
$$

Recursively solving the last system leads to the required result.

The distribution $\psi_{x}(k)$ is then determined by differentiation of the GF,

$$
\psi_{x}(k)=\left.\frac{1}{k !} \frac{d^{k}}{d z^{k}} \tilde{\psi}_{x}(z)\right|_{z=0} .
$$

Theorem 8. The mean number of switches $\mathbb{E}[K]$ of the repair facility can be calculated by

$$
\begin{gathered}
\mathbb{E}[K]=1+\sum_{i=0}^{L-N_{2}-1} C_{N_{2}}^{N_{2}+i-1} v_{N_{2}+i}, \text { where } \\
C_{n}^{l}=\prod_{k=n}^{l} \tau_{k}, \tau_{N_{1}+1}=\frac{\left(N_{1}+2\right) \lambda}{\left(N_{1}+2\right) \lambda+\mu}, v_{N_{1}+1}=\frac{\mu}{\left(N_{1}+2\right) \lambda+\mu}, \\
\tau_{k}=\frac{(k+1) \lambda}{(k+1) \lambda+\mu-\mu \tau_{k-1}}, v_{k}=\frac{\mu v_{k-1}}{(k+1) \lambda+\mu-\mu \tau_{k-1}}, N_{1}+2 \leq k \leq L-1, k \neq N_{2}, \\
\tau_{N_{2}}=\frac{\left(N_{2}+1\right) \lambda}{\left(N_{2}+1\right) \lambda+\mu-\mu \tau_{N_{2}-1}-v_{N_{2}-1}}, v_{N_{2}}=\frac{(24)}{\left(N_{2}+1\right) \lambda+\mu-\mu \tau_{N_{2}-1}-v_{N_{2}-1}},
\end{gathered}
$$

Proof. The conditional moments of the number of switches are calculated by

$$
\bar{\psi}_{x}=\left.\frac{d}{d z} \tilde{\psi}_{x}(z)\right|_{z=1}
$$

Obviously $\mathbb{E}[K]=\bar{\psi}_{(0,0)}$. The last value can be obtained from the system

$$
\left.\bar{\psi}_{x}=\sum_{\substack{y \neq x \\ y \neq\left(1, N_{2}\right)}} \frac{\lambda_{x y}}{\lambda_{x}} \bar{\psi}_{y} 1_{\left\{x \neq\left(0, N_{2}-1\right)\right.}\right\}+\frac{N_{2} \lambda}{\lambda_{x}}\left(\bar{\psi}_{\left(1, N_{2}\right)}+1\right) 1_{\left\{x=\left(0, N_{2}-1\right)\right\}}
$$

obtained by differentiating of (22) over $z$ at point $z=1$. 
(a)

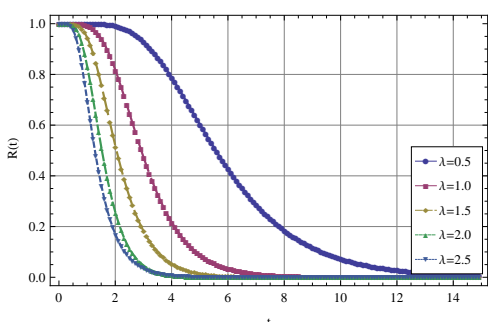

Fig. 1 Function $R(t)$ versus $\lambda$ (a) and $\mu$ (b) (a)

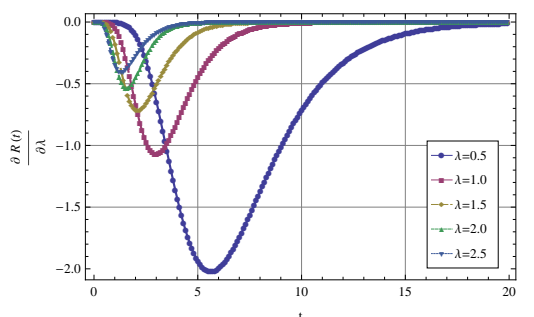

(b)

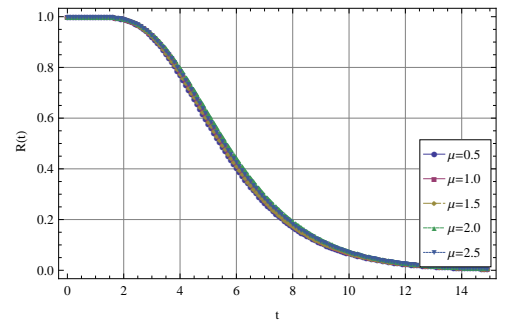

(b)

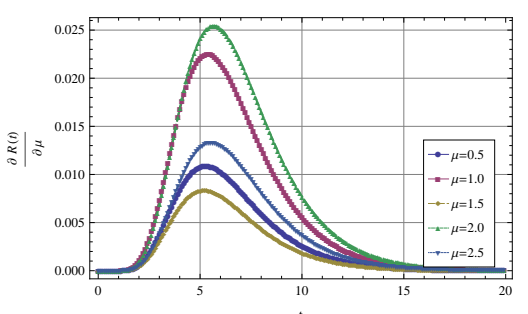

Fig. 2 Sensitivity of $R(t)$ versus $\lambda$ (a) and $\mu$ (b)

(a)

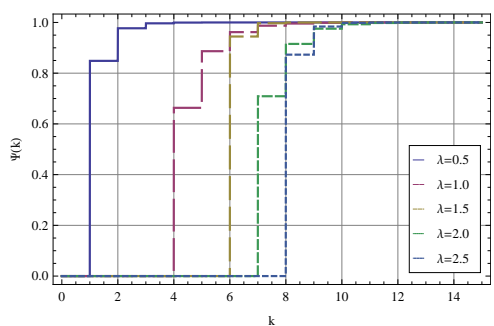

(b)

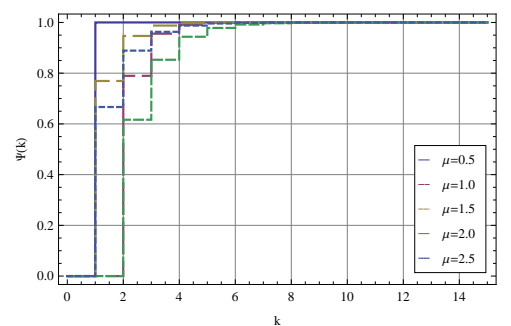

Fig. 3 Function $\Psi(k)$ versus $\lambda$ (a) and $\mu$ (b)

\section{Sensitivity analysis}

Here we perform a sensitivity analysis for changes in the reliability function $R(t)$ together with changes of specific values of system parameters, e.g. failure intensity $\lambda$ and repair intensities $\mu$. We differentiate the expression (13) and get

$$
\frac{\partial R(t)}{\partial \lambda}=-\frac{\partial \pi_{(1, L)}(t)}{\partial \lambda}, \frac{\partial R(t)}{\partial \mu}=-\frac{\partial \pi_{(1, L)}(t)}{\partial \mu} .
$$


All examples are calculated for the optimal hysteresis policy $\left(N_{1}, N_{2}\right)$, which maximizes the total average reward $g$ during the life time. For the inversion of the LPs a numerical method is used. Further we fix $L=10, c_{1}=0.5, c_{2}=0.1, c_{3}=c_{4}=1.5$, and consider two cases:

1. $\lambda$ is varied from 0.5 to 2.5 with a lag 0.5 and $\mu=2.5$,

$$
\begin{aligned}
\mathbb{E}[T] & =\{6.01 ; 3.14 ; 2.22 ; 1.64 ; 1.42\}, \\
\left(N_{1}, N_{2}\right) & =\{(7,9) ;(5,6) ;(1,4) ;(2,3) ;(0,2)\}
\end{aligned}
$$

2. $\mu$ is varied from 0.5 to 2.5 with lag 0.5 and $\lambda=0.5$,

$$
\begin{aligned}
\mathbb{E}[T] & =\{5.88 ; 5.97 ; 5.92 ; 6.10 ; 6.01\}, \\
\left(N_{1}, N_{2}\right) & =\{(3,9) ;(7,8) ;(8,9) ;(7,8) ;(7,9)\} .
\end{aligned}
$$

Figures illustrate the function $R(t)$ with derivatives as well as the the discrete distribution function $\Psi(k)=\sum_{i=1}^{k} \psi(i)$. It is observed that the reliability function is more sensitive to parameter changing in case $\lambda<\mu$, otherwise the sensitivity almost vanishes. Interesting observations have been made also for the distribution function $\Psi(k)$ of the number of switches $K$.

Acknowledgements This work was funded by the Russian Foundation for Basic Research, Project No. 16-37-60072 mol_a_dk, supported by the Austro-Hungarian Cooperation Grant No. 90öu6, OMAA 2014, Stiftung Aktion Österreich-Ungarn.

\section{References}

1. C.-H. Wu and J.-C- Ke: Computational algorithm and parameter optimization for a multiserver system with unreliable servers and impatient customers. Journal of Computational and Applied Mathematics, 235 (2010), 547-562.

2. D. Efrosinin: Optimal parameters of the degrading unit with state-dependent repair time. Procceedings of the MMR2013, Stellenbosch (2013).

3. V.A. Kopnov: Optimal degradation processes control by two-level policies. Reliability Engineering and System Safety, 66 (1999), 1-11.

4. R.K. Mallik: On the solution of a second order linear homogeneous difference equation with variable coefficients. Journal of Mathematical Analysis And Applications, 215 (1997), 32-47.

5. D.N.P. Murphy and B.P. Iskandar: A new shock damage model: Part II - Optimal maintenance policies. Rel. of Eng. System Safety, 31 (1991), 211-231.

6. V. Rykov and D. Efrosinin: Degradation models with random life resources. Communications in statistics-Theory and Methods, 39 (2010), 398-407 .

7. V. Rykov and D. Efrosinin: On optimal control of systems on their life time. Recent Advances in System Reliability (Springer, 2011), 307-319.

8. T.M. Welte, J. Vatn and J. Heggset: Markov state model for optimization of maintenance and renewal of hydro power components. Proceedings of the 9th International Conference PMAPS, Stockholm (2006). 